\title{
Chemifluorescent Optical Fiber Immunosensor for On-Site Bioaerosol Monitoring System
}

\author{
Koji Toma ${ }^{1}$, Yurika Suzuki ${ }^{2}$, Chisato Kishikawa ${ }^{2}$, \\ Mana Saito ${ }^{3}$, Kumiko Miyajima ${ }^{1,4}$, Takahiro Arakawa ${ }^{1}$, \\ Hiroji Shimomura $^{3}$ and Kohji Mitsubayashi ${ }^{1,2, *}$ \\ 'Department of Biomedical Devices and Instrumentation, \\ Institute of Biomaterials and Bioengineering, Tokyo Medical and Dental University, \\ 1-5-10 Kanda-Surugadai, Chiyoda-ku, Tokyo 101-0062, Japan \\ ${ }^{2}$ Graduate School of Medical and Dental Sciences, Tokyo Medical and Dental University, \\ 1-5-45 Yushima, Bunkyo-ku, Tokyo 113-8549, Japan \\ ${ }^{3}$ Faculty of Health Science Technology, Bunkyo Gakuin University, \\ 1-19-1 Mukogaoka, Bunkyo-ku, Tokyo 113-8668, Japan \\ ${ }^{4} \mathrm{Japan}$ Society for the Promotion of Science, \\ 5-3-1 Kojimachi, Chiyoda-ku, Tokyo 102-0083, Japan
}

(Received May 27, 2015; accepted September 15, 2015)

Key words: optical fiber, immunoassay, chemifluorescence, layer-by-layer assembly

In this work, we describe an optical fiber immunosensor for on-site bioaerosol monitoring application. Considering its integration to the monitoring system, a polymethyl methacrylate (PMMA) fiber was used as a sensor probe. The fiber edge surface was modified with a layer-by-layer (LbL) assembled film for immobilizing primary antibodies. The LbL film was formed by depositing alternating layers of cationic and anionic polymers, namely, poly(allylamine hydrochloride) (PAH) and poly(acrylic acid) (PAA), respectively. To assess the sensor performance, a model immunoassay experiment was conducted with mouse $(\mathrm{mIgG})$ and horseradish peroxidase (HRP)-labeled anti-mouse (a-mIgG) antibodies. mIgG was immobilized on PAAs in the LbL film. After affinity binding of HRP-labeled a-mIgG to $\mathrm{mIgG}$, a solution containing a fluorescent substrate, 10-acetyl-3,7-dihydroxy-phenoxazine (ADHP), is added. The resultant fluorescent product, resorufin, is then excited with light of $570 \mathrm{~nm}$ wavelength from the fiber edge, and the emitted fluorescence of $600 \mathrm{~nm}$ wavelength is collected by an identical fiber edge and detected by a photomultiplier tube. As results of the experiment, a sensor limit of detection (LOD) of $85 \mathrm{ng} \cdot \mathrm{mL}^{-1}$ and a dynamic range of $0.71-100 \mu \mathrm{g} \cdot \mathrm{mL}^{-1}$ for a-mIgG were determined. It was demonstrated that the optical fiber immunosensor has superiority in size and flexibility with high sensor performance, and thus can be easily incorporated into a monitoring system.

*Corresponding author: e-mail: m.bdi@tmd.ac.jp 


\section{Introduction}

A bioaerosol is a suspension of airborne particles containing substances of biological origin or living organisms. Exposure to bioaerosol relates to a wide range of health issues such as infectious diseases, allergies or acute toxic effects. ${ }^{(1)}$ For example, influenza is a respiratory infectious disease with symptoms including fever and physical weariness. Worldwide, approximately 100 million people are infected each year, and in some severe cases, it causes death. ${ }^{(2)}$ Currently, influenza virus infection is mainly diagnosed by detecting the virus from a sampled specimen such as nasal discharge and pharynx wiping liquid by methods including immunochromatographic assay, enzymelinked immunosorbent assay (ELISA), and real-time polymerase chain reaction (PCR). ${ }^{(3-7)}$ That is, the current medical testing relies on the specimen sampled from a patient who may already have been infected, and thus is not suitable for preventing infection.

The main infection routes for influenza include droplet infection and droplet nuclei infection. ${ }^{\left({ }^{8-12}\right)}$ For droplet infection, virus-containing droplets released from an infected individual via coughing or sneezing directly reach the mucous membrane of the nose or throat, and for droplet nuclei infection, the nucleus of the droplet remains in the air for a long time after the water of the droplet has dried out. Therefore, it is of great importance to monitor the amount of airborne viruses and its change with time on-site to prevent from coming into contact with them. In order to do so, a system that collects airborne viruses and quantifies the amount on-site, i.e., an on-site monitoring system, is useful. Recently, we have developed a bioaerosol sampler in which collected airborne substances are transported from the gas phase to the liquid phase through porous membranes. ${ }^{(13)}$ To achieve on-site monitoring, the system needs to be capable of both sampling the airborne substances and measuring them; thus, the bioaerosol sampler and an immunosensor are to be installed in the system. In immunosensors, an optical fiber is widely used, and some plastic optical fibers show superiority in workability and surface modifications. ${ }^{(14-20)}$ Furthermore, by conducting the immunoassay on a fiber edge and placing the fiber edge in close proximity to the gas-liquid interface in the bioaerosol sampler, the detection time could be shortened, which is important for simultaneous sampling and measurement.

In this work, we aim to develop an optical fiber immunosensor on whose surface immunoassay is performed. First, we developed a technique to immobilize primary antibodies on the polymethylmethacrylate (PMMA) fiber edge surface by employing a layer-by-layer (LbL) method with a cationic polymer poly (allylamine hydrochloride) (PAH) and an anionic polymer poly (acrylic acid) (PAA). Then, a model immunoassay experiment was conducted to assess the sensor performance. In the immunosensor, the detection of target molecules relies on chemifluorescence.

\section{Materials and Methods}

\subsection{Reagents and chemicals}

PAH [15000 molecular weight (MW)], PAA (45000 MW), N-(3dimethylaminopropyl)- $N$ ' -ethylcarbodiimide hydrochloride (EDC), $\mathrm{N}$-hydroxysuccinimide (NHS), 2,2'-azino-bis(3-ethylbenzothiazoline-6-sulfonic acid) 
diammonium salt (ABTS), and ethanolamine were all purchased from Sigma-Aldrich. Hydrogen peroxide $\left(\mathrm{H}_{2} \mathrm{O}_{2}\right)$ was purchased from Kanto Kagaku. Mouse IgG and horseradish peroxidase (HRP)-labeled mouse IgG-Fab fragment antibody (a-mIgG) were from Bethyl Laboratories. A chemifluorescent HRP substrate kit (QuantaRed) containing 10-acetyl-3,7-dihydroxy-phenoxazine (ADHP), an enhancer solution, a stable peroxide solution, and a stop solution was from Thermo Fisher Scientific. Bovine serum albumin (BSA) was from Itoham Foods. Phosphate-buffered saline-Tween (PBS-T) solution was prepared by adding $0.05 \%$ Tween 20 to PBS (10 mM phosphate buffer, $140 \mathrm{mM} \mathrm{NaCl}, 3$ $\mathrm{mM} \mathrm{KCl}, \mathrm{pH}$ 7.4). A chromogenic substrate solution for absorbance measurement with both the optical fiber immunosensor and ELISA was prepared by mixing $30 \% \mathrm{H}_{2} \mathrm{O}_{2}$ and $0.05 \%(\mathrm{w} / \mathrm{v})$ ABTS in citrate phosphate buffer $(70 \mathrm{mM}, \mathrm{pH} 4.2)$ at a volume ratio of $1 / 1000$.

\subsection{Immunosensor apparatus}

An optical fiber (diameter $=2 \mu \mathrm{m}$, Asahi Kasei) whose core is made of PMMA was chosen as a sensor probe owing to its material superiority in probe fabrication, incorporation into the on-site monitoring system, and optical properties. The fiber edge surface was polished with a polisher (Rev, Krell Technologies) and three different polishing films. First, the surface was roughly polished with a silica film (particle diameter $=30 \mu \mathrm{m}$ ) followed by wet polishing with a finer silica film (particle diameter $=$ $9 \mu \mathrm{m}$ ). Finally, mirror finishing was applied to the surface with an alumina film (particle diameter $=0.3 \mu \mathrm{m})$. After polishing, the PMMA fiber was connected to a bifurcated optical fiber (diameter $=600 \mu \mathrm{m}, \mathrm{BIF} 600-\mathrm{VIS} / \mathrm{NIR}$, Ocean Optics $)$ at its common end [see Fig. 1(a)]. Excitation light emitted from a green light-emitting diode (LED, $\lambda=535 \mathrm{~nm}$, Brightek Optoelectronics) is coupled to one leg of the bifurcated fiber. The light was guided to the PMMA fiber edge and emitted to excite the fluorescent product resorufin (absorption wavelength $\lambda_{\mathrm{ab}}=570 \mathrm{~nm}$, emission wavelength $\lambda_{\mathrm{em}}=585 \mathrm{~nm}$ ). Here, resorufin was produced as a result of the enzymatic reaction between ADHP and HRP as illustrated in Fig. 1(b). The emitted fluorescence was collected from an identical fiber edge, then transmitted through the other leg of the bifurcated fiber to a photomultiplier tube (PMT, H7421-40, Hamamatsu Photonics). In the setup, two different band-pass filters (BPs, Asahi Spectra) were placed after the LED $(570 \pm 10 \mathrm{~nm})$ and before the PMT $(600 \pm 10 \mathrm{~nm})$ to eliminate unwanted light. The optical property of the PMMA fiber was examined and compared with that of a glass optical fiber (F1000-900 PROBE, Ocean Optics) by using a resorufin solution. Both the PMMA and glass fibers showed similar output dependences on the resorufin concentration in the range of $0.1-50 \mu \mathrm{M}$, which indicates that the prepared PMMA fiber can be an alternative to the glass fiber.

\subsection{Primary antibody immobilization on PMMA fiber edge surface by LbL method}

The LbL method was adopted to immobilize a primary antibody on the PMMA fiber edge surface because it is built by a self-assembly process by the electrostatic force of oppositely charged layers, and allows easy attachment of desirable functional groups on the sensor surface to immobilize primary antibodies. ${ }^{(21-25)}$ Furthermore, a PMMA 


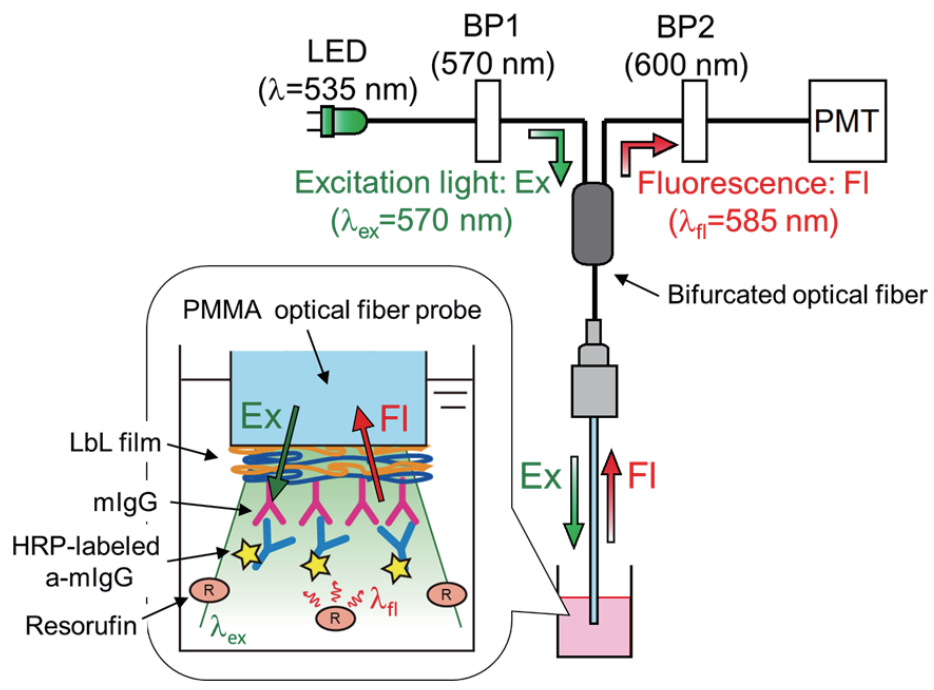

(a)

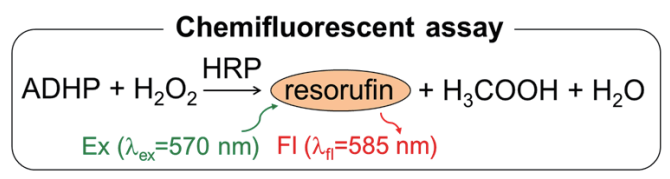

(b)

Fig. 1. (Color online) (a) Schematic diagram of the sensor setup. Excitation light of $570 \mathrm{~nm}$ wavelength is illuminated from a PMMA optical fiber probe and emitted fluorescence of 585 $\mathrm{nm}$ wavelength from resorufin is collected from an identical fiber. (b) Reaction scheme of HRPmediated enzymatic reaction to produce resorufin.

fiber tends to be negatively or positively charged depending on the environment of use, e.g., solution $\mathrm{pH}$; thus, it is easy to form an $\mathrm{LbL}$ film on the fiber. Cationic $\mathrm{PAH}$ and anionic PAA were separately dissolved in $\mathrm{NaCl}$ solution $(0.5 \mathrm{M}, \mathrm{pH} 4.0)$ at 2 and $1 \mu \mathrm{g} \cdot \mathrm{mL}^{-1}$, respectively. Figure 2(a) shows the procedure of LbL film formation. A negatively charged PMMA fiber was firstly immersed in the PAH solution, then it was rinsed with ultrapure water. Subsequently, the same procedure was conducted for the PAA solution. After repeating the deposition process several times (PMMA fiber/[PAH/ $\mathrm{PAA}]_{N} ; N$ is the number of repetitions for [PAH/PAA] alternating layers), the primary antibody $\mathrm{mIgG}$ was covalently bonded to PAA layers through EDC/NHS coupling [see Fig. 2(b)]. Carboxylic groups of PAA layers were activated in a mixture of EDC and NHS solutions (each concentration in ultrapure water $=0.1 \mathrm{M}$; incubation time $=30 \mathrm{~min}$ ), followed by rinsing with ultrapure water. Then, it was reacted with $\mathrm{mIgG}$ dissolved in PBS-T for $1 \mathrm{~h}$. Finally, unreacted active esters of the carboxylic groups 


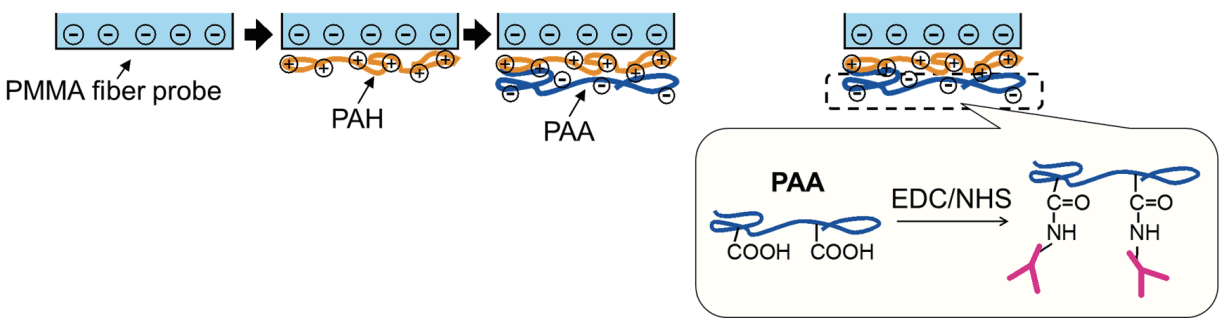

(a)

(b)

Fig. 2. (Color online) (a) Layer-by-layer (LbL) film formation on the edge surface of PMMA fiber probe with alternating layers of cationic PAH and anionic PAA. (b) EDC/NHS coupling between PAA and $\mathrm{mIgG}$ for immobilizing $\mathrm{mIgG}$ on the fiber edge surface.

were passivated by $30 \mathrm{~min}$ incubation in ethanolamine ( $1 \mathrm{M}, \mathrm{pH} 8.5)$, and afterwards, the PMMA fiber was immersed in $1 \mathrm{wt} \% \mathrm{BSA} / \mathrm{PBS}-\mathrm{T}$ solution to further prevent nonspecific binding.

\subsection{Model immunoassay experiment with ELISA}

As a control, ELISA was used to measure a-mIgG. First, $10 \mu \mathrm{g} \cdot \mathrm{mL}^{-1} \mathrm{mIgG}$ in carbonate-bicarbonate buffer solution $(40 \mathrm{mM}, \mathrm{pH} 9.6)$ was added and incubated for $20 \mathrm{~h}$ at $4{ }^{\circ} \mathrm{C}$ on a plastic microtiter plate. The well was then incubated with $0.1 \%(\mathrm{w} /$ w) bovine serum albumin in PBS-T (blocking buffer) for $1 \mathrm{~h}$ at room temperature so as to reduce nonspecific bindings. After the addition of HRP-labeled a-mIgG in PBS-T at a given concentration, the well was incubated for $1 \mathrm{~h}$. The well was rinsed with PBS-T three times at the end of each step. Finally, 0.05\% ABTS solution was added to produce blue colored products through the HRP-mediated enzymatic reaction, followed by stopping the reaction with $1 \%(\mathrm{w} / \mathrm{v})$ SDS in ultrapure water. The absorbance of the colored solution at $405 \mathrm{~nm}$ wavelength was measured with a microplate reader (SH1000Lab, Corona Electric).

\section{Results and Discussion}

\subsection{Assay optimization with PMMA cuvette}

First, the layer numbers of PAH/PAA in the LbL film and $\mathrm{mIgG}$ concentrations in the immunoassay were optimized with PMMA cuvettes. In contrast to the PMMA fiber, the cuvette was positively charged; therefore, the first layer in the LbL film was anionic PAA. After the same LbL film formation and $\mathrm{mIgG}$ immobilization procedures on the cuvette bottom surface as those for the PMMA fiber (PMMA cuvette/PAA/[PAH/PAA $]_{N} / \mathrm{mIgG}$ ), the cuvette was kept filled with $5 \mu \mathrm{g} \cdot \mathrm{mL}^{-1} \mathrm{HRP}$ labeled a-mIgG solution in PBS-T for $1 \mathrm{~h}$ so that a-mIgG bound to $\mathrm{mIgG}$. Afterwards, 
the same procedures as those for the aforementioned experiment using ELISA, in which a blue-colored product through the enzymatic reaction with HRP was produced and its absorbance at $405 \mathrm{~nm}$ wavelength was measured, were performed. Figure 3(a) shows the absorbance with different layer numbers on the PMMA cuvette surface. When only cationic PAH was deposited on the PMMA cuvette, the absorbance was similar to that for the bare cuvette. This is attributed to the inefficient formation of the PAH layer on the cuvette owing to the electrostatic repulsive force between them and the fact that no carboxylic group exists in $\mathrm{PAH}$; thus, $\mathrm{mIgG}$ did not form a covalent bond. In contrast, when a single PAA layer was formed on the cuvette, the absorbance increased by a factor of about three as there are electrostatic attractive forces between the PAA and the cuvette, and the covalently bonded $\mathrm{mIgG}$. The absorbance further increased until $N=2$ (PMMA cuvette/PAA/[PAH/PAA $]_{2} / \mathrm{mIgG}$ ), and then dropped when $N=3$. This sudden decrease may be explained by the charge imbalance that could induce the LbL film defects; therefore, an insufficient amount of primary IgG was immobilized. The maximum increase at $N=2$ was by a factor of 18 with respect to that of the bare cuvette. These results indicate that using $\mathrm{LbL}$ with $N=2$ and a covalent bond for the primary $\operatorname{IgG}$ immobilization allows one to achieve the primary IgG immobilization on the PMMA fiber edge surface with the largest amount and a strong binding.

Next, the amount of primary antibody that was added for the immobilization was optimized by varying the $\mathrm{mIgG}$ concentration from 2 to $25 \mu \mathrm{g} \cdot \mathrm{mL}^{-1}$, as shown in Fig. 3(b). The absorbance increased with increasing $\mathrm{mIgG}$ concentration and then became saturated. Since little change was observed at concentrations over $10 \mu \mathrm{g} \cdot \mathrm{mL}^{-1}, 10$ $\mu \mathrm{g} \cdot \mathrm{mL}^{-1} \mathrm{mIgG}$ was used in the subsequent experiments.

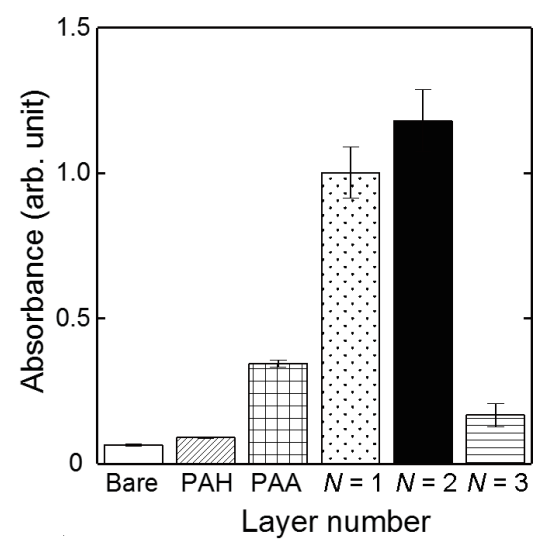

(a)

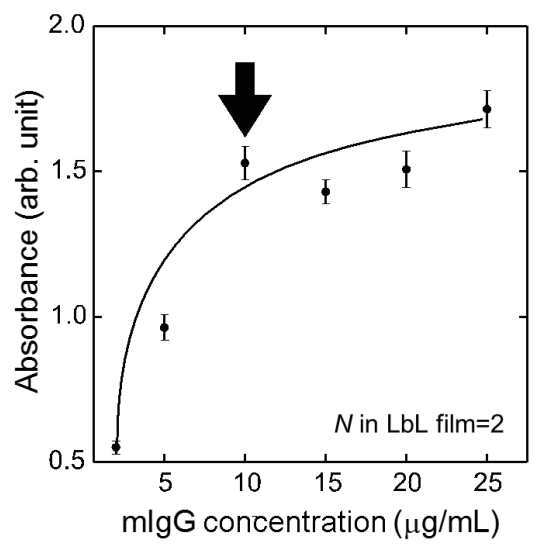

(b)

Fig. 3. (a) Effect of LbL films on the absorbance. $N$ denotes the number of repetitions for [PAA/ $\mathrm{PAH}]$ alternating layers. PAH or PAA indicates that only a single layer is deposited on the fiber surface. (b) Effect of the added mIgG concentration on absorbance for LbL film with $N=2$. 
The selectivity of the immunoassay was also evaluated. Figure 4 shows the changes in absorbance for the $5 \mu \mathrm{g} \cdot \mathrm{mL}^{-1} \mathrm{a}-\mathrm{mIgG}$ and rabbit $\mathrm{IgG}$ (rIgG, Sino Biological). Owing to the high specificity of a-mIgG to $\mathrm{mIgG}$ and the anti-fouling property of the LbL layers with BSA blocking, little adsorption of $\mathrm{rIgG}$ was observed. This experiment demonstrated that the immunoassay with the LbL film can be used to selectively detect an antigen, e.g., an influenza virus.

\subsection{Model immunoassay experiment with PMMA optical fiber immunosensor}

To characterize the optical fiber immunosensor, a model immunoassay experiment was performed with $\mathrm{mIgG}$ and a-mIgG. On the negatively charged edge surface of the PMMA optical fiber, an LbL film was formed by starting with cationic PAH deposition. The number of layers $N=2$ for the LbL film was chosen as optimized above: PMMA fiber/[PAH/PAA $]_{2}$. Afterwards, the carboxylic groups of PAA layers were activated with EDC/NHS, followed by the addition of $10 \mu \mathrm{g} \cdot \mathrm{mL}^{-1} \mathrm{mIgG}$ and its covalent bonding. After passivation with ethanolamine and rinsing, different concentrations of HRP-labeled a-mIgG were added and incubated for $1 \mathrm{~h}$ to bind to $\mathrm{mIgG}$ on the fiber edge. Here, although the same incubation time of a-mIgG as that for ELISA was chosen, it could be shortened with further optimizations. The fiber was then immersed into a well that was filled with a mixture of the enhancer and the stable peroxide solutions at $50 \mu \mathrm{L}$ each. After $30 \mathrm{~s}$ of incubation, a fluorescent substrate molecule, $1 \mu \mathrm{L}$ of ADHP, was added to start the enzymatic reaction with HRP, then the excitation light was illuminated to excite the produced fluorescence molecules of resorufin. The emitted fluorescence was simultaneously collected from an identical fiber, and the intensity was measured. The time-dependent intensity changes at various a-IgG concentrations are shown in Fig. 5(a). After adding the fluorescent substrate ADHP, an increase in intensity and its dependence

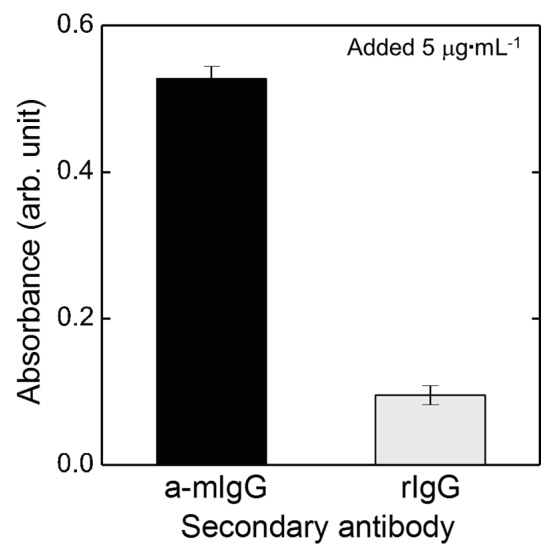

Fig. 4. Selectivity assessment of the immunoassay. $\operatorname{rgG}$ was used as a control, whereas a-mIgG was affinity-bound to $\mathrm{mIgG}$ on the LbL film. 


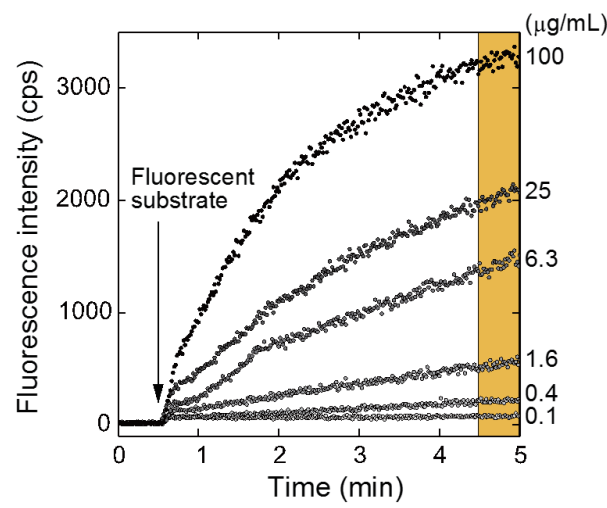

(a)

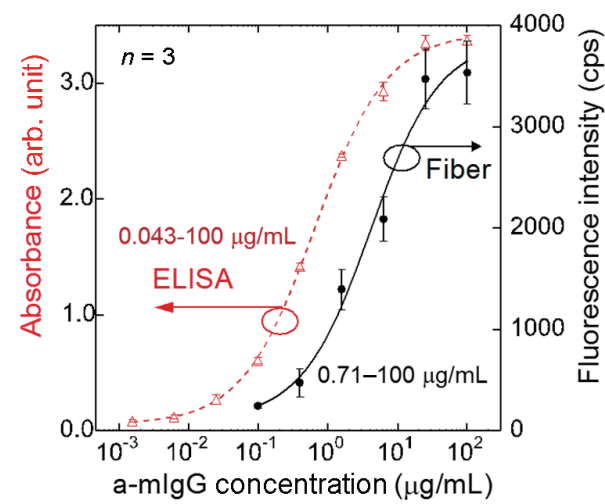

(b)

Fig. 5. (Color online) (a) Time-dependent fluorescence intensity change for various a-mIgG concentrations after adding the fluorescent substrate ADHP. (b) Calibration curves for the optical fiber immunosensor and ELISA (control) for a-mIgG.

on the concentration were observed. The resultant calibration curve is shown in Fig. 5(b). The plotted fluorescence intensities were obtained by averaging it for the last $30 \mathrm{~s}$ in Fig. 5(a). The plots can be described using the following equation with a correlation coefficient of 0.992 :

$$
\text { Fluorescence intensity }(\mathrm{cps})=A+(B-A) /\left(1+\left([\mathrm{a}-\mathrm{mIgG}] / C^{D}\right)\right. \text {, }
$$

where $A=112, B=3860, C=4.14$, and $D=0.879$ are the coefficients, and [a-mIgG] is the concentration of added a-mIgG in $\mu \mathrm{g} \cdot \mathrm{mL}^{-1}$. The limit of detection (LOD) or the dynamic range of the sensor was determined at the concentration where three or ten times the standard deviation of the blank $(\sigma=77.0 \mathrm{cps})$ equals the fluorescence intensity. It resulted in the developed sensor LOD of $85 \mathrm{ng} \cdot \mathrm{mL}^{-1}$ and the dynamic range of $0.71-100$ $\mu \mathrm{g} \cdot \mathrm{mL}^{-1}$. For comparison, the same model immunoassay experiment was performed using ELISA, and the result is together shown in Fig. 5(b). ELISA showed LOD of 5.2 $\mathrm{ng} \cdot \mathrm{mL}^{-1}$ and the dynamic range of $0.043-100 \mu \mathrm{g} \cdot \mathrm{mL}^{-1}$. Although ELISA shows about one order of magnitude better LOD or dynamic range, it is not suited for the on-site monitoring system because of its long measurement time (about two days from $\mathrm{mIgG}$ immobilization to the detection of a-mIgG) and the large device size. The developed optical fiber has superiority in measurement time (about a few hours from LBL film formation to the detection), size, and flexibility; therefore, it is time-saving and easier to incorporate the fiber to the system. Besides, the capture antibodies are immobilized on the fiber edge, which allows one to bring the sensor surface in close proximity to the gas-liquid interface in the on-site monitoring system and possibly to shorten the time for antigen-antibody binding events in the liquid phase; thus, monitoring could be achieved. 


\section{Conclusions}

In this paper, we described a PMMA optical fiber immunosensor for on-site monitoring application. The sensor takes advantage of two characteristics. First, immunoassay on the fiber edge could shorten the time of antigen-antibody binding by approaching the fiber to the gas-liquid phase in the monitoring system where the collected bioaerosol is carried into the liquid phase. Second, the use of PMMA as an optical fiber material makes the sensor flexible and easy to incorporate into the monitoring system. The primary antibody was immobilized on the edge surface by the LbL technique with PAA and PAH. After optimizing the layer numbers in the LbL film and the primary antibody concentration, model immunoassay experiments with $\mathrm{mIgG}$ and HRP-labeled a-mIgG were performed to assess the sensor performance. The fluorescent resorufin molecules produced through the HRP-mediated enzymatic reaction were illuminated from the fiber with excitation light of $570 \mathrm{~nm}$ wavelength. Simultaneously emitted fluorescence of $585 \mathrm{~nm}$ wavelength was collected from an identical fiber, and then its intensity was measured to quantify the a-mIgG concentration. The sensor LOD and dynamic range were determined as $85 \mathrm{ng} \cdot \mathrm{mL}^{-1}$ and $0.71-100$ $\mu \mathrm{g} \cdot \mathrm{mL}^{-1}$, respectively. These experiments demonstrated that the developed optical fiber immunosensor has superiority in size and flexibility with high sensor performance. In the future, we anticipate the combination of the optical fiber immunosensor with a bioaerosol-collecting device to develop an on-site monitoring system.

\section{Acknowledgements}

This work was supported by the Japan Society for the Promotion of Science (JSPS) Grants-in-Aid for Scientific Research System, Japan Science and Technology Agency (JST), and Ministry of Education, Culture, Sports, Science and Technology (MEXT) Special Funds for Education and Research "Research Promotion of Neo-Biology".

\section{References}

1 J. Douwes, P. Thorne, N. Pearce and D. Heederik: Ann. Occup. Hyg. 47 (2003) 187.

2 N. Sriwilaijaroen and Y. Suzuki: Proc. Jpn. Acad. Ser. B 88 (2012) 226.

3 G. Cattoli, A. Drago, S. Maniero, A. Toffan, E. Bertoli, S. Fassina, C. Terregino, C. Robbi, G. Vicenzoni and I. Capua: Avian Pathol. 33 (2004) 432.

4 A. C. M. Boon, A. M. F. French, D. M. Fleming and M. C. Zambon: J. Med. Virol. 65 (2001) 163.

5 Q. He, S. Velumani, Q. Du, W. L. Chee, K. N. Fook, R. Donis and J. Kwang: Clin. Vaccine Immunol. 14 (2007) 617.

6 N. J. Montalto and D. O. Robert: Am. Fam. Physician 67 (2003) 111.

7 K. A. Poehling, M. R. Griffin, R. S. Dittus, Y.-W. Tang, K. Holland, H. Li and K. M. Edwards: Pediatrics 110 (2002) 83.

8 C. J. Roy and D. K. Milton: N. Engl. J. Med. 350 (2004) 1710.

9 W. G. Lindsley, F. M. Blachere, R. E. Thewlis, A. Vishnu, K. A. Davis, G. Cao, J. E. Palmer, K. E. Clark, M. A. Fisher, R. Khakoo and D. H. Beezhold: PLoS One 5 (2010) e15100. 
10 D. A. Edwards, J. C. Man, P. Brand, J. P. Katstra, K. Sommerer, H. A. Stone, E. Nardell and G. Scheuch: Proc. Natl. Acad. Sci. U.S.A. 101 (2004) 17383.

11 P. Fabian, J. J. McDevitt, W. H. DeHaan, R. O. P. Fung, B. J. Cowling, K. H. Chan, G. M. Leung and D. K. Milton: PLoS One 3 (2008) e2691.

12 D. K. Milton, M. P. Fabian, B. J. Cowling, M. L. Grantham and J. J. McDevitt: PLoS Pathog. 9 (2013) e1003205.

13 K. Miyajima, Y. Suzuki, D. Miki, M. Arai, T. Arakawa, H. Shimomura, K. Shiba and K. Mitsubayashi: Talanta 123 (2014) 241.

14 D. J. Monk and D. R. Walt: Anal. Bioanal. Chem. 379 (2004) 931.

15 M. D. Marazuela and M. C. Moreno-Bondi: Anal. Bioanal. Chem. 372 (2002) 664.

16 P. Leung and T. F. George: Phys. Rev. B 36 (1987) 4664.

17 T. Konry, A. Novoa, S. Cosnier and R. S. Marks: Anal. Chem. 75 (2003) 2633.

18 J. Zubia and J. Arrue: Opt. Fiber Technol. 7 (2001) 101.

19 G. Wandemur, D. Rodrigues, R. Allil, V. Queiroz, R. Peixoto, M. Werneck and M. Miguel: Biosens. Bioelectron. 54 (2014) 661.

20 K. Miyajima, G. Itabashi, T. Koshida, K. Tamari, D. Takahashi, T. Arakawa, H. Kudo, H. Saito, K. Yano, K. Shiba and K. Mitsubayashi: Environ. Monit. Assess. 182 (2011) 233.

21 F. N. Crespilho, V. Zucolotto, O. N. Oliveira and F. C. Nart: Int. J. Electrochem. Sci. 1 (2006) 194.

22 M. Michel, V. Toniazzo, D. Ruch and V. Ball: ISRN Mater. Sci. 2012 (2012) 1.

23 J. Dai, G. L. Baker and M. L. Bruening: Anal. Chem. 78 (2006) 135.

24 S. Derveaux, B. G. Stubbe, C. Roelant, M. Leblans, B. G. De Geest, J. Demeester and S. C. De Smedt: Anal. Chem. 80 (2008) 85.

25 H. Shen, J. Watanabe and M. Akashi: Anal. Chem. 81 (2009) 6923. 